Himalayan J. Soc. Sci. \& Humanities ISSN: 0975-9891

Vol. 14, (2019) 51-57 DOI: https://doi.org/10.51220/hjssh.v14i1.8

\title{
Critical Study Of Literature Written By “Kavi Ratna” Sri Sri Krishna Semwal
}

\author{
Madan Mohan Nauriyal \\ Lecturer, Government Intermediate College Parsundakhal, District Pauri Garhwal, Uttarakhand
}

*Corresponding Author Email: madanmohan20863@gmail.com

Received: 22.10.2019; Revised: 07.11.2019; Accepted: 28.11.2019

CSociety for Himalayan Action Research and Development

\begin{abstract}
In the present communication a critical study is presented on the literature written by Sri Sri Kreshna Semwal. He has composed various poems combining his devotional expressions, satirical, eclectic, character based and emotional sources."Bhakti Rasamritam Stuti Kavya" composed by Sri Sri Kreshna Semwal is filled with the extracts of basis devotion of indian culture. This is a unique collection of devotional songs. In this, there is an incarnation of Kavya Ras from the worship of different Gods and Goddesses. The poet has composed poetry on devotional expressions as well as on real subjects. On the one hand the poet has written the adornment of Shani, in other he tried to highlight the evil practices of the society by writing "Bhimshatakam". His composition "Indirakeertishatakam" is based on the life of former Prime Minister smt Indira Gandhi. It is tried to analyse the all faces of writings of Sri Sri Kreshna Semwal in this presentation.
\end{abstract}

Keywords: Literture, Sri Sri Kreshna Semwal, Analysis

\section{कविरत्न श्री श्रीकृष्ण सेमवाल विरचित साहित्य का समीक्षात्मक अध्ययन}

\section{मदन मोहन नौड़ियाल}

\section{प्रवक्ता इण्टर कालेज परसुण्डाखाल जिला पौड़ी गढ़वाल}

*Corresponding Author Email: madanmohan20863@gmail.com

Received: 22.9.2019; Revised: 7.10.2019; Accepted: 28.10.2019

CSociety for Himalayan Action Research and Development

सारांश

प्रस्तुत शोध पत्र में कविरत्न श्री श्रीकृष्ण सेमवाल की साहित्यिक कृतियों का समीक्षात्मक अध्ययन प्रस्तुत किया गया है। आपने भक्तिभाव, व्यंगमूलक, अन्योपदेषभूत, चरित तथा स्तोत्र भाव के मूलाश्रित विभिन्न काव्यों की रचना की है। भक्तिरसामृतम् स्तुति काव्य कविरत्न श्री श्रीकृष्ण सेमवाल द्वारा विरचित रचना भारतीय संस्कृति की आधारभूमि भक्ति 
रस से ओतप्रोत है। यह संग्रह भक्ति गीतों का अनूठा संग्रह है। इसमें विभिन्न देवी देवताओं की स्तुति से काव्य रस की अवतारणा हुई है। भक्तिभाव के साथ-साथ कवि ने वास्तविक विषयों पर काव्य रचना की। जहां एक ओर शनिसमाराधना लिखी वहीं दूसरी ओर भीमशतकम् लिखकर समाज में फैली कुरीतियों को दूर करने का प्रयास किया। आपकी कृतिया साहित्य की विशिष्टता के साथ-साथ सामाजिक मूल्यों का भी प्रसार करती हैं और उनमें अभिव्यक्त सूक्तियां व्यक्ति को सन्मार्ग की ओर ले जाती है। आपकी कृति इन्दिरा कीर्तिशतकम इन्दिरा गांधी के व्यक्तित्व पर आधारित है। प्रस्तुत शोध पत्र में यथासंभव कविरत्न श्री श्रीकृष्ण सेमवाल द्वारा विरचित साहित्य की समीक्षा करने का प्रयास किया गया है।

कुंजी शब्द : कविरत्न श्री श्रीकृष्ण सेमवाल, साहित्यिक कृतियां, भक्तिरसामृतम, शनिसमाराधना, भीमशतकम, अनुरक्ति पीयूषम, समीक्षात्मक अध्ययन

कविरत्न श्रीकृष्ण सेमवाल का जम्न 05 जनवरी 1944 को चमोली जिले के हयूण गांव में हुआ था। आपने भक्तिभाव, व्यंगमूलक, अन्योपदेषभूत, चरित तथा स्तोत्र भाव के मूलाश्रित विभिन्न काव्यों की रचना की है। प्रस्तुत शोध पत्र में उनकी इन सभी कृतियों का समीक्षात्मक अध्ययन प्रस्तुत किया गया है।

कविरत्न श्रीकृष्ण सेमवाल ने स्तोत्र साहित्य को एक विशिष्ट आयाम तक पहुंचाया है। इसी परिप्रेक्ष्य में भक्तिरसामृतम् स्तोत्र काव्य का अवतरण हुआ है। भक्तिरसामृतम् स्तुति काव्य कविरत्न श्री श्रीकृष्ण सेमवाल द्वारा विरचित रचना भारतीय संस्कृति की आधारभूमि भक्ति रस से ओतप्रोत है। यह संग्रह भक्ति गीतों का अनूठा संग्रह है। इसमें विभिन्न देवी देवताओं की स्तुति से काव्य रस की अवतारणा हुई है। आठ खण्डो में वर्णित यह खण्ड काव्य ईष प्रार्थना से प्रारम्भ है।

समे: सम्भूय संयाम:

प्रभो गीतानिगायाम:

ईष प्रार्थना में कवि का कथ्य है कि हम अज्ञानी बालक हैं। हे प्रभो हमे सन्मार्ग की ओर प्रेरित कीजिये। तदुपरान्त कवि ने सरस्वत वन्दना की है कवि दण्डी के अनुसार "मानसे रमतां नित्यं सर्वषुक्ला सरस्वती" इस रचना में कविरत्न श्रीकृष्ण सेमवाल ने धर्म दर्षन, साहित्य, संगीत, ज्ञान भक्ति, कला आदि बौद्धिक सुसंस्कारों क माता के रूप में सरस्वती वन्दना की है।

धमार्थ चिन्तन संरते कविता कला कलितेष्वरी

संगीत गीति सुगायिनी प्रतिभा प्रदान परायणें

हंसानने सुविराजते सकलाश्रये जनमोददे

मातः सरस्वति पाहि मां शरणागतं शरण प्रदे

इनकी इस रचना में ईश्वर के अतिरिक्त बटुक भैरव के रूप में हनुमान जी की स्तुति तथा मां जगजननी के विविध आयामों की महिमा का वर्णन किया गया है। भक्ति रसामृतम् में विभिन्न छन्दों में प्रार्थनायें संकलित हैं तथा पूर्ण आत्म निवेदन का भाव है। इस कृति में ईष प्रार्थना तथा सरस्वती शीर्षक स्तुतियां मात्र चार-चार श्लोकों मे है। ग्रन्थ का मंगलाचरण रूप प्रारम्भ में उपन्यस्त है। हनुमत्सुप्रभातम में 51 पद्य, वर्णात्मक भगवती सुप्रभातम् में 51 पद्य, कामना मे 51 पद्या, सर्वमंगलाषतकम् में 101 पद्य काव्यायिनी स्वतः में 21 पद्य तथा ज्वाल्पा देवी स्तवः में 15 पद्य हैं। भक्ति रसामृतम् एक कोष काव्य है। यह काव्य एक ही विधा से ओतप्रोत है किन्तु एक दूसरे से पृथक अनेक स्तोत्रों का समन्वित रूप है।

संस्कृत साहित्यकारों ने काव्य के प्रमुख रूप से दो भेद माने हैं। उनमें से श्रव्य काव्य एक भेद है। अनेक कवियों ने श्रीमद्भागवत में वर्णित कथाओं तथा देवीभागवत में वर्णित कथाओं को श्रव्य काव्यों का उपजीव्य बनाया है। इन सभी कवियों ने अपने काव्य में युगानुरूप परिवर्तन किये, जिसकी प्रत्यक्ष छाप इनके काव्यों में दृष्टव्य है। 
संस्कृत महाकाव्यों के बाद खण्डकाव्यों की परम्परा आती है। इसी परम्परा के अन्तर्गत पुष्पदन्त के दो खण्डकाव्य नागकुमार चरित ${ }^{2}$ और यषोधर चरित ${ }^{3}$, सुकुमार का कृष्ण विलास ${ }^{4}$, रामभद्र का पतंजलि चरित ${ }^{5}$, विष्वनाथ का राघव विलास ${ }^{6}$, सोमेष्वर का रामषतक ${ }^{7}$ आदि खण्ड काव्य हैं। भक्तिरसामृतम् भी एक खण्ड काव्य है। यह खण्डकाव्य आठ खण्डों में विभक्त है। इस खण्ड काव्य में भक्ति का अजस्र स्रोत प्रस्फुटित होता है। इस प्रकार की काव्य रचना विरल ही उपलब्ध होती है।

भक्तिभाव के साथ-साथ कवि ने वास्तविक विषयों पर काव्य रचना की। जहां एक ओर शनिसमाराधना लिखी ${ }^{8}$ वहीं दूसरी ओर भीमशतकम्श लिखकर समाज में फैली कुरीतियों को दूर करने का प्रयास किया। कविरत्न सेमवाल की कृतियों को चार भागों में बांटा जा सकता है। कुछ काव्य व्यंग्यमूलक, कुछ काव्यों को अन्योपदेष भूत तथा अन्य को हम चरित पर आधारित काव्य तथा पुनः अन्य को हम स्तोत्र काव्य कह सकते हैं। कविरत्न के काव्य, साहित्य की विशिष्टता के साथ-साथ सामाजिक मूल्यों का भी प्रसार करते हैं और उनमें अभिव्यक्त सूक्तियां व्यक्ति को सन्मार्ग की ओर ले जाती है। इन्होने राजनैतिक व्यक्तित्व पर भी कृतियां लिखी हैं। इन्दिरा कीर्ति शतकम्म 1973 मे लिखा जो इन्दिरा प्रियदर्शिनी (इन्दिरा गांधी) के व्यक्तित्व पर आधारित है।

यावच्चन्द्र दिवाकरौ विलसतौ यावच्च वायु स्तथा

यावद्व्योम विराजते जननि हे यावद्धरा शोभते

यावद्विष्वमिदं विभाति सकलं यावज्जलं निर्मलं

तावत्कीर्तिरतीव भव्य विमला ते राजतां भ्राजताम्

कहीं पर वे अपने काव्य में इन्दिरा को देवी मानकर लिखते है-

भुवनपालने विष्णुरूपिणी

लयकर क्षणे रूद्ररूपिणी

अबल रक्षणे मातृरूपिणी

जयतु सेन्दिरा चित्ररूपिणी

अनुरक्ति पीयूषम11 मे कविरत्न सेमवाल प्रेम की परिभाषा इस प्रकार देते हैं-

न हि वाच्छति यः प्रियः कदा

निजसर्वस्व समर्पितप्यहो

प्रतिदान फलम प्रिया प्रति

जगति प्रेम तदैव कथ्यते

अपनी कृति विन्सर महादेव स्तोत्ररत्नावलि ${ }^{12}$ में कविरत्न सेमवाल लिखते हैं-

सघनवन निवासी लास्य लीला विलासी

भुवनमय विनाषी विन्सरस्याधिवासी

कठिन कृति विकासी श्री महादेव एष:

वितरतु भुवनेस्मिन सर्वदा सुप्रभातम्

वहीं शनिसमाराधना ${ }^{13} 2003$ मे प्रकाषित कृति भगवान सूर्य के पुत्र शनि के विषय में लिखते है। भले ही ज्योतिषशास्त्र में शनि को क्रूर ग्रह माना गया हो फिर भी शनि से बड़ा कृष्णभक्त आज तक नहीं हुआ।

कृष्णभक्तं तपोमूर्ति ज्ञानिनं शान्तमानसम्

प्रणमामि सदा भक्त्या सूर्यपुत्र शनैष्चरम्

समाज में फैली कुरीतियों, जातिप्रथा, छुआछूत को भी कवि ने 1991 में प्रकाशित रचना भीमषतकम्स मे कुप्रथाओं को दूर करने का स्तुत्य प्रयास किया है। इस रचना में गौतम बुद्ध को प्रणाम करते हुए अस्पृष्यता के विषय में श्रीकृष्ण सेमवाल लिखते हैं-

सर्पादयो विषयुक्ता भुवि जन्तवो ये 
सिंहादि हिंसनपरा पषवस्समस्ता

एतेस्पृषा: किल च सन्ति न वा मनुष्या:

किन्नौ बुधै: किमपि तब विचारितं कदा

वही अपनी कृति संघेषक्ति कलौयुगे 15 में देषभक्त श्री बलिराम केशवराम हेडगेवार का अभ्युदय व संघ के क्रियाकलापों का वर्णन कविरत्न सेमवाल ने निम्नवत् किया है।

रामस्य कार्याय तदत्र पुर्प्या, प्राणर्पणं सेवक वृन्दवन्धे

यै यै कृत तान्प्रति भक्ति भावेः श्रद्धांजलिः सादरमर्पयाम:

अपनी कृति "वाग्वैभव"16 में सूक्तियों का समावेष करते हुए कविरत्न लिखते हैं-

दिवसस्य विभूषणं रविः

सुनिषायाष्च विभूषणं विधू

कमलं च हृदस्य भूषणं

सुजनो हास्ति विभूषणं भुव:

103 श्लोक हैं-

"हिमाद्रि पुत्राभिनन्दन काव्यम्" 17 हेमवती नन्दन बहुगुणा के जीवनवृत्त पर आधारित कृति है। इस काव्य में

प्रदेषोधुनापि स्मरत्युत्तरस्थः

त्वदीयं प्रियं मुख्यमन्त्रित्वकालम

यदा सर्व लोकस्सुखेः पूर्णआसी

च्चरित्रेण वित्तेन सत्कर्मणापि

वहीं भक्ति रसामृतम् में अन्तरयामी ईष्वर के विषय में लिखते हैं-

महान्त, सृष्टिकर्तारं मुनीनां चिन्तना धारम्

गुणानां सागरं वन्ध विभुषषणमं समायाम:

इसके अतिरिक्त कविरत्न सेमवाल ने स्फुट कवितायें भी लिखी हैं। यथा-वालः, बालचर्या, मदीय कामना क्षेत्रम् गंगा, कवि, मति, भानु, धेनु, देवी, पिता, माता, प्रष्नोत्तराणि विद्यालय चर्या, निर्देषा, ख्यात संख्या, जयतात प्रिय भारतवर्षधरा आदि। नारी केवल पालन करने वाली ही नहीं बल्कि सम्पूर्ण ब्रह्माण्ड का आधार है। सरस्वती वीणा वादिनी, विद्याधिष्ठात्री अज्ञान नाषिनी, शुभ्रवस्तधारिणी हंसासने सुविराजते भवबन्धन का नाष करने वाली है। यह श्री सेमवाल की छन्द अलंकारों से युक्त विभिन्न अवसरों पर गायी जाने वाली सुन्दर कृति है। इनके द्वारा सुविरचित हनुमत्सुप्रभातम् प्रातः काल में पढ़ा जाने वाला स्तोत्र है। इनकी भक्ति से संसार की समस्त बाधायें दूर हो जाती है।

प्रयान्ति वाधाः मनुजस्य सर्वाः

पवित्रा पुण्य स्मरणेन यस्य

स माननीयो हनुमान मदीय:

करोतु नित्यं मम सुप्रभातम्

51 पद्यो में रचित हनुमत्सुप्रभातम् ${ }^{18}$ मे कवि ने हनुमान का शौर्यपूर्ण गुणगान किया है।

मुखेपि रामो, हृदयेपि रायो, विभाति कार्येष्वपियस्मरामः

कपीष्वरो राममयः स एव करोतु नित्यंमम सुप्रभातम्

हनुमत्सुप्रभातम् को हृदयंगम करने पर विदित होता है कि काव्यरचना पर तुलसीदास रचित रामचरित मानस, वाल्मीकिय रामायण, हनुमानबाहुक तथा श्रीमद्भागवत महापुराण के किम्पुरूषवर्ष का कवि पर स्पष्ट प्रभाव परिलक्षित होता है। शक्तिपूजा के क्रम में वर्णात्मक भगवतीसुप्रभातम् 51 पद्यों में श्रीकृष्ण सेमवाल विरचित दुर्गतिनाषिनी भगवती दुर्गा का भक्तिभाव से र्मरण किया गया है। पद्यों को पढ़ने से प्रतीत होता है कि श्रीकृष्ण 
सेमवाल पर दुर्गा सप्तषती के सिद्ध कुंजिका स्तोत्र का प्रभाव अवष्य पड़ा है। माता दुर्गा कलिकल्मष नाषिनी, ऋषिमुनियों द्वारा पूजित देवगणों की आश्रय स्थली, लृतन्त्र में देवमाता, श्री तन्त्र में माया तथा माहेष्वर सूत्र ऋलृक में शारदा है। भगवती मंगलमयी है। डिंच सूत्र भी भगवती के वर्णात्मक विचित्र रूप को प्रकट करता है। मां दीन दुखियों की रक्षक है।

त्रस्तस्य या शरणदा सदयाश्रयाया

या भामिनी व्यथित मानव मोदिनीय:

या पालिनी ननु भुवो जगदीष्वरी सा

नित्यं करोतु भुवनेयम सुप्रभातम्

कामना खण्ड में कविरत्न श्रीकृष्ण सेमवाल कहते हैं कि आदि शक्ति की पूजा षोडषोपचार पूजा(पाद्य, अर्ध्य, धूप, दीप, नैवेद्य, सदक्षिणा) से मानस पूजा की जाती है। भक्त चाहे कितना ही सामर्थ्यवान हो वह पूजा की विषाल सामग्री नहीं जुटा पाता फिर भी भक्त की कामना होती है कि वह दुर्लभ से दुर्लभ पूजा भगवती के चरणों में समर्पित कर सके।

यथा-

द्राक्षा फलानि कदलीदल दाडिमानि

खर्जूर जम्बु सुरसाल फलादि कानि

लेकोपचार चरितस्तव पुत्रकोयं

तुभ्यं प्रदास्यति च सर्वफल प्रदात्रयै

श्रीकृष्ण सेमवाल कामना खण्ड में भगवती से मानवीय मूल्यों के संरक्षण की कामना करते हैं-

घोरे महाकलियुगे जगदम्ब पश्य

भष्टाष्च: चरित्राहनने पटव कृतहना:

टाचारपूतचरितं परितापयन्ति

मिथ्याकथा प्रकथनै: किमुनात्रकष्टम्

सर्वमंगलाषतकम् खण्ड में कवि ने 100 पद्यों में भगवती का मंगलगान किया है-

महित मंगल मंगल मंगले

कमलं निर्मल निर्मल निर्मले

जन विनोद विनोद विनोदिते

विजयसे जयदे जयतेजिते

कवि ने सबकी शरणदात्री भगवती को ही माना है-

न हि बलं न धनं न कुलं गृहं

न च सरवा न हि कोपि च बाधव:

न च मतिर्नगतिर्नरतिर्र में

त्वमयि केवल मेव ममाम्बिके

काव्यायिनी स्तव: खण्ड में काव्याधन ऋषि की तपस्या से प्रसन्न होकर छठी देवी दुर्गा पुत्रीरूप में काव्यायिनी कहलायी। वृन्दावन में गोपियों ने श्रीकृष्ण को पतिरूप में पाने के लिए मार्गषीर्ष के महीने यमुना में तपस्या की थी। श्रीकृष्ण सेमवाल ने 21 श्लोकों में कात्यायनी देवी की सुन्दर स्तुति की है। कात्यायनी भगवती ही पर्वतराज कन्या पार्वती है। जैसा कि वर्णन किया गया है-

शिवा त्वं काली त्वं कर धृत कपालिन्यसि शुभे

भवानी त्वं देवी भुवन जननी त्वं गिरिसुता

विशाला कल्याणी त्वमसि जनवाणी जयकरा 
त्वमेव क्लानं मां सुखय भुवि कात्यायिनी मुदा

ज्वाल्पा देवी भुविभगवती भासतां में सुमाता खण्ड में कवि के अनुसार पौराणिक अनुश्रुतियों के अनुसार जब महाकाली ने राक्षसों का संहार किया तब कालीमठ में ज्वाल्पा देवी विराजमान हुई। कवि ने ज्वाल्पा व धारी देवी को एक ही माना है-

धारी देवी धृतिमतिमयी धर्मदा धैर्यदात्री

धूम्राक्षादि प्रबलवलिनो धूर्तदैत्य प्रहर्त्री

या माता मामवति नितरां स्नेह भावेन भूमौ

ज्वाल्पा देवी त्वमसि किलसामन्मतेष्चिन्तनेन

संक्षेप में हम कह सकते हैं कि भक्ति रसामृतम् देवी की स्तुति मे लिखा गया एक स्तोत्र काव्य है। यथाकालीरूपो ललित ललिते दिव्य लक्ष्म्यास्वरूपे

विद्यादेवी द्रुहिण गृहणी रूपतोप्येवनित्यम्

या माता में वसति भुवने दिव्य कालीमठेस्मिन

ज्वाल्पा रूपेप्यवतु जननि प्रत्यंह मां सुतं सा

आचार्य विष्वनाथ के साहित्य दर्पण की कसौटी पर यह खण्डकाव्य खरा उतरता है।

खण्डकाव्यम् भवेत्काव्यसैयन देषानुसारिच

आचार्य भरत ने रसनिष्पति के सन्दर्भ में विभानुभाव व्यभिचारि संयोगाद्रसनिष्पत्ति माना है। भक्तिरसामृतम् को हम भक्ति रस कह सकते हैं। आचार्य भरत ने काव्य के दस गुण माने हैं-श्लेष, प्रसाद, समता, समाधि, माधुर्य, ओज, पद सौकुमार्य, अर्थ, उदारता, कान्ति। ये सभी गुण भक्ति रसामृतम् में दिखायी देते हैं। कविरत्न श्रीकृष्ण सेमवाल ने काव्यादर्ष के रचयिता दण्डी के मानसे रमतां नित्यं सर्वषुक्ला सरस्वती के आधार पर सरस्वती की स्तुति की है। सरस्वती को ग्यारह रूद्र, आठ वसु, वारह आदित्य, विष्वेदेवा अग्नि, इन्द्र वरूण मित्र सभी को धारण करने वाली बताया है। "हे ज्ञान दे वरदे प्रिये विद्या विभाविभुवल्ल में साहित्य वारिधि में अनुप्रास व रूपक की छटा दृष्टव्य है। काव्यतत्व के आधार पर सरस्वती वन्दना में सत्वगुण हैं। शब्दषक्ति के आधार पर अमिधा शब्द शक्ति है। हनुमत्सुप्रभातम् उपेन्द्रव्रजा छन्द में विरचित है। निषाचराचार विनाष दक्षः में अनुप्रास अलंकार है। हनुमान के स्वभाव का वर्णन करने में कवि द्वारा रूपक अलंकर की छटा दृष्टव्य है।

समस्तं विष्वस्य विकासकर्तु:

प्रभोर्गुणानां पद पंकजानाम्

वहीं वर्णात्मक भगवती सुप्रभातम् खण्ड में विभिन्न वर्णों की वर्णानुवृत्ति द्वारा अनुप्रास अलंकार की छटा बिखेरी है। यथा-

लम्बोदरी सुललना ललित त्वमेव, लोक प्रियापि लवणापि मनोहरात्वम्

उक्त स्तोत्र के सभी श्लोक बसन्ततिलका छन्द मैं है, कामना खण्ड में कवि द्वारा शार्दूल विक्रीडित छन्द में मंगलाचरण की परम्परा का निर्वहन किया है यथा-

विष्वढ्यां विष्ववन्धां त्रिभुवन जननी तापसन्तापहन्ती

धर्माख्या धर्मरूपा धृतिमति महितां मन्दातानाषकत्रीम्

सर्वोकृष्टां सुमान्यां सकलसुखकरीं सर्वसम्पत्प्रदात्री

ध्यायेहं ध्यानगम्यां त्रिपुरविजयनीम्बिका विष्वधात्रीम्

सर्वमंगलाषतकम खण्ड में 101 श्लोक हैं। पहले से 40 श्लोक तक द्रुतविलम्बित छन्द में है। 41 से 60 तक षिखरिणी छन्द में 61 से 100 तक द्रुत विलम्बित छन्द मे हैं। इस शतक मे कवि ने यमक, अनुप्रास, उपमा व रूपक अलंकारों का वर्णन किया है। 101 वां श्लोक अनुष्टुप छन्द मे है यथा-

मुखरता मयि यद्यपि विद्यते 
सकलतापहरे सदयाम्बिके

अयमहन्तु तथापि भवाम्युमे

सपदि मूक समस्तव सम्मुखे

कात्यायिनी स्तव खण्ड पढ़ने से करूणा का भाव होने के कारण करूणरस की अनुभूति होती है। कहीं-कहीं काव्य में रूपक की छटा भी दृष्टव्य है।

तथापि त्वत्पादम्बुज पतन मृगोह्यनुचर कात्यायिनी स्तव के श्लोक क्षिते क्षेमार्थ या नगपतिसुता दिव्यवन्दना मे अमिधा शब्दषक्ति है। निष्कर्ष रूप में सम्पूर्ण काव्य में अनुप्रास रूपक उपमा आदि अलंकारों का सुन्दर चित्रण है। सभी श्लोकों में प्रसाद गुण है जिसके आधार पर कहा जा सकता है कि भक्तिरसामृतम् एक सफल स्तोत्र काव्य है।

\section{संदर्भ ग्रन्थ}

1 भक्तिरसामृतम्, कविरत्न श्रीकृष्ण सेमवाल, श्री रामजी लाल अग्रवाल, बनारसीदास इस्टेट, तिमारपुर, 1996

2 नागकुमार चरित, पुष्पदंत कवि, 1996

3 यषोधर चरित, पुष्पदंत कवि, 2004

4 कृष्ण विलास, कृष्ण दास, प्रचारणी सभा नई दिल्ली

5 पतंजलि चरित, राम चंद्र दीक्षित

6 राघव विलास, आचार्य विश्वनाथ

7 रामशतक, कविरत्न श्रीकृष्ण सेमवाल, श्री रामजी लाल अग्रवाल, बनारसीदास इस्टेट, तिमारपुर, 2003

8 शनिसमाराधना, उपरोक्त

9 भीमशतकम, कविरत्न श्रीकृष्ण सेमवाल, श्री रामजी लाल अग्रवाल, बनारसीदास इस्टेट, तिमारपुर, 1991

10 इन्दिरा कीर्ति शतकम, उपरोक्त 1973

11 अनुरक्ति पीयूषम, उपरोक्त 2000

12 विन्सर महादेव स्तोत्ररत्नावलि, उपरोक्त 2000

13 शनिसमाराधना, श्रीकृष्ण सेमवाल, श्री रामजी लाल अग्रवाल, बनारसीदास इस्टेट, तिमारपुर, 2003

14 भीमषतकम, कविरत्न श्रीकृष्ण सेमवाल, श्री रामजी लाल अग्रवाल, बनारसीदास इस्टेट, तिमारपुर,1991

15 संघेषक्ति कलौयुगे, कविरत्न श्रीकृष्ण सेमवाल, श्री रामजी लाल अग्रवाल, बनारसीदास इस्टेट, तिमारपुर, 2000

16 वाग्वैभव, उपरोक्त 2000

17 हिमाद्रि पुत्राभिनन्दन काव्यम, उपरोक्त 2000

18 हनुमत्सुप्रभातम, उपरोक्त 1996 